\title{
¿Para quién trabajan los derechos humanos? Críticas al sistema internacional de los derechos humanos desde una perspectiva interseccional
}

\author{
Who do Human Rights work for? Criticism of the international \\ human rights system from an intersectional perspective
}

\author{
Magdalena Abarca Lizana \\ Universidad de Chile
}

\begin{abstract}
RESUMEN La finalidad de este artículo es dar cuenta de un conjunto de críticas teóricas y prácticas en torno al sistema internacional de derechos humanos desde una perspectiva integral. Esto se hará tomando en cuenta enfoques de género, raciales y provenientes de países no hegemónicos ni occidentales. Además, se llevará a cabo un análisis de los derechos humanos en distintas facetas y en distintos momentos, lo que revelará problemáticas consustanciales del discurso de los derechos humanos y problemas contingentes de su proyecto práctico. Para esto se revisarán algunos casos específicos en que el actuar de los organismos que componen el sistema de derechos humanos ha sido contrario a lo que se predica en el discurso. Se concluye que es necesario replantear las bases del sistema de derechos humanos, ya que de lo contrario seguirán sirviendo para perpetuar el statu quo y no ayudarán a quienes lo necesitan, ya que los problemas actuales son una consecuencia lógica de la forma en que fueron formulados.
\end{abstract}

PALABRAS CLAVE Sistema internacional de los derechos humanos, críticas desde la interseccionalidad, perspectiva de género.

ABSTRACT This article pursues to review different critics, both theoretical and practical, towards the international human rights system from an intersectional perspective, considering gender, racial and non-western approaches. Many aspects and stages of human rights will be analyzed, showing that while there are current issues regarding human rights as a practical project, there also are many inherent issues regarding its narrative itself. This will be illustrated by revising some particular cases in which international agencies that are part of the international human rights system have acted against what human rights, in theory, promote. It is concluded that it is strictly necessary to reevaluate the grounds of the international human rights system as a whole, other- 
wise human rights will keep availing and legitimizing the status quo, not helping the ones who need them the most, because current world issues are a logical consequence of the way the system was (and is) structured.

KEYWORDS International human rights system, intersectional criticism, gender perspective.

\section{Introducción}

Hay muchos momentos que pueden ser considerados históricos. Uno de ellos ocurrió el 10 de diciembre de 1948, cuando la Asamblea General de las Naciones Unidas aprobó la Declaración Universal de los Derechos Humanos. Esto marcó un hito en la época, especialmente si se tiene en cuenta que recientemente se había terminado la Segunda Guerra Mundial, en la cual se cometieron un sinnúmero de crímenes que en la actualidad son catalogados como de lesa humanidad. El objetivo de esta declaración fue marcar un precedente, es decir, que los horrores cometidos en ambas guerras mundiales fueran algo que nunca se repitiera.

Para lograrlo era necesario entender que existían ciertos derechos que eran intrínsecos a la naturaleza humana, inalienables e iguales para todos. Se trata, entonces, de un piso mínimo (Anyangwe, 1996). El objetivo de este documento internacional declarativo adoptado de manera unánime por los países que formaban parte de las Naciones Unidas fue establecer los derechos humanos básicos en treinta artículos. Considerando que este no era un documento vinculante (Anyangwe, 1996: 83), se entendió que a través de otros tratados internacionales que sí lo fueran se desarrollarían y agregarían diversos derechos humanos. Así, cada país que firmase y ratificase el tratado se encontraría obligado a cumplirlo. En resumen, así es como se empieza a formar el sistema internacional de los derechos humanos.

No obstante, es evidente que esto no se cumplió. Actualmente es difícil hablar de derechos que sean inherentes al hombre, inalienables y para todos por igual. Esto ocurre por varios factores, partiendo por el hecho de que en realidad los derechos humanos no pueden ser definidos como aquellos que pertenecen a los seres humanos (García, 1989). Se suman a lo anterior diversas condiciones: la diferencia entre la teoría y la práctica en el ámbito de los derechos humanos, y la situación actual de tensión constante entre homogeneidad y diversidad que se vive en el mundo (tendiendo los derechos humanos a lo neutral), entre otros. Así, se puede percibir que los derechos humanos no son lo que en algún minuto se propuso que fueran.

En la actualidad no solo se ha vuelto una necesidad formular una crítica a los derechos humanos, sino también a todo su sistema, porque en realidad los derechos humanos son un movimiento, una ideología y una teoría: no son simples derechos 
(Arias, 2015). Al poder reconocerlos como un movimiento resulta lógico que detrás de ellos haya una idea política y social.

A pesar de esto, la única forma de saber quiénes crearon los derechos humanos, para qué fueron creados y a quiénes les servirán es a través de una teoría crítica que resulta especialmente necesaria si se atiende a las esperanzas que se depositaron en ellos. Con las respuestas de dichas preguntas es posible vislumbrar una serie de críticas que sirven para formar paulatinamente una teoría crítica integral sobre los derechos humanos, que pueda otorgar los lineamientos sobre su futuro tomando en cuenta a todos los seres humanos, incluyendo a quienes han sido dejados de lado por su movimiento político y social.

En ese sentido, este trabajo pretende estructurar una crítica desde una perspectiva interseccional, lo que implica, según se verá más adelante, tomar en cuenta los diversos factores de discriminación existentes que se ven prolongados $-\mathrm{y}$ que son ocultados- por el sistema actual de derechos humanos.

Así, en primer lugar, se entregarán ciertas nociones previas que servirán para comprender el objetivo del trabajo: una conceptualización de lo que realmente son los derechos humanos, la definición de interseccionalidad y por qué ella nos sirve para la teoría crítica de los derechos humanos, y los tres ejes centrales a partir de los cuales se realizarán las críticas. Posteriormente, estas últimas se dividirán en dos secciones principales: las que guardan relación con la creación de los derechos humanos en cuanto sistema o estructura, y las que guardan relación con el funcionamiento actual de dicho sistema. Si bien las críticas serán principalmente teóricas, habrá ciertas referencias a la situación práctica del sistema internacional de los derechos humanos, el cual también puede ser fuertemente criticado desde una perspectiva internacional.

Finalmente, se desarrollará una conclusión a partir de lo anterior, proponiendo que en realidad son «estos» derechos humanos los que no resultan útiles para constituir un cambio real en la situación de todas y cada una de las personas. Esto se debe a que fueron hechos a la medida de quienes eran - y siguen siendo- lo suficientemente poderosos como para instaurarlos, lo que no impide que los derechos humanos puedan ser repensados en clave interseccional (lo cual sin duda requiere de un movimiento social y político fuerte y constante) para que puedan considerarse realmente universales.

\section{Definiciones previas}

¿Qué son los derechos humanos? Entre la teoría y el movimiento político-social

Los derechos humanos son mucho más que simples derechos. Por una parte, son un discurso que en tanto propuesta teórica cuenta con una estructura específica que se sustenta en premisas valóricas específicas, y que sirve para legitimar diversas 
posturas y acciones (Arias y Ponte, 2015). Por otra parte, son un proyecto práctico (Arias y Ponte, 2015: 13) porque persiguen un cambio real en la sociedad que refleja la propuesta teórica. Siguiendo esta última definición, también pueden ser concebidos como un movimiento político y social porque los derechos humanos se han transformado en una bandera de lucha para grupos importantes de la sociedad (Gallardo, 2006). Finalmente, también pueden ser concebidos como valores históricos, ya que representan un «lugar común» en un momento determinado (García, 1989: 95), es decir, se trataría de puntos de consenso en un contexto determinado.

En resumen, los derechos humanos son multifacéticos, ya que pueden ser entendidos de diversas formas según la perspectiva desde la que se observen. Es necesario tenerlo en cuenta porque las distintas críticas que se realicen pueden enfocarse más en uno de los significados de los derechos humanos que en otros, especialmente en cuanto pueden proponerse críticas prácticas y críticas teóricas, no obstante, la necesidad de una relegitimación de ambos aspectos (Arias 2015: 18).

\section{¿Qué es la interseccionalidad?}

La interseccionalidad puede ser definida como:

Una herramienta para el análisis y la elaboración de políticas, que aborda múltiples discriminaciones y nos ayuda a entender la manera en que conjuntos diferentes de identidades influyen sobre el acceso que se pueda tener a derechos y oportunidades (Symington, 2004).

En ese sentido, la interseccionalidad funciona como instrumento teórico y metodológico que sirve para dar cuenta de diversas relaciones de poder desde una percepción cruzada de los diferentes factores de identidades que se presentan oprimidas, como el género, la etnia, la clase social, la raza e incluso la ciudadanía. Por ejemplo, si bien ser mujer implica un factor identitario que implica opresión, no estará en la misma posición una mujer caucásica heterosexual y de clase media que una mujer de raza negra bisexual o lesbiana (Smith, 200o). El ejemplo anterior da cuenta de cómo funciona la interseccionalidad: se hace cargo de todos esos factores. De esta forma, es útil a la hora de poner en marcha una teoría crítica, ya que permite abarcar diversos factores, evaluando cómo cada uno de ellos se ve afectado por una misma institución (en este caso, el sistema de derechos humanos), y a su vez mostrando con cuánta intensidad afecta a cada uno de ellos y a todos esos factores reunidos en una misma persona. En consecuencia, si bien los derechos humanos fueron creados por hombres que reflejaron sus preocupaciones principales en ellos (Facio, 2000) y por eso afectan a las mujeres en general, es cierto que lo hacen de manera especial a las mujeres musulmanas, ya que los derechos humanos fueron pensados únicamente desde la perspectiva occidental (dominante) y cristiana (Bunch, 1990). En este caso, 
el desarrollo de las luchas en nombre de los derechos humanos afecta a las mujeres de forma desigual, según su posición cultural y económica en el contexto global, ya que los derechos humanos en tanto movimiento han mantenido un sesgo político hegemónico hasta el día de hoy.

Finalmente, una teoría crítica de los derechos humanos debe partir desde la interseccionalidad para ser integral porque de otra forma se corre el riesgo de resituar ciertas identidades en la oscuridad, lo que conlleva a la desprotección y contraría el objetivo de estas críticas: replantear los derechos humanos para que sean realmente universales.

\section{Críticas teóricas en cuanto a la creación del sistema de derechos humanos}

Los derechos humanos como proyecto práctico y propuesta teórica se encuentran en crisis. Debido a esto, necesitan relegitimarse. Sin embargo, es posible - y necesariohacer una crítica que ponga énfasis en su momento y forma de creación, es decir, que ponga en duda los cimientos mismos en que se apoyan. En realidad, es posible que los derechos humanos hayan nacido en crisis y eso es precisamente lo que se explorará en esta sección.

¿Quiénes crearon el sistema internacional de los derechos humanos?

Como se señaló anteriormente, los derechos humanos son mucho más que derechos, ya que son un movimiento y un discurso (Arias 2015: 13) que se encuentra en función de los intereses de un cierto grupo de personas (Howse 2002). Fueron creados como derechos a modo de respuesta a las preocupaciones de quienes los crearon, quienes en su mayoría fueron hombres caucásicos heterosexuales (Charlesworth, Chinkin y Wright, 1991), jerarquizándolos (García, 1989: 94). Esto es lógico si se entiende que los derechos humanos también son un movimiento político y social, en el que quienes luchan lo hacen según sus propias preocupaciones. Un ejemplo claro de lo anterior es el hecho de que fuesen mujeres como Eleanor Roosevelt las que lucharon por la inclusión de la proscripción de discriminación por sexo en la Declaración Universal de los Derechos Humanos (Wiesen, 1992). Roosevelt, como mujer, luchó por plasmar preocupaciones específicas de un determinado grupo social en los derechos humanos.

Ciertamente, el concepto de derechos humanos no es estático y no le pertenece, en teoría, a ningún grupo en especial, sino que se expande en tanto han ido cambiando las necesidades y aspiraciones de los protagonistas del movimiento de los derechos humanos (Bunch, 1990: 487). El problema es que, en la práctica, desde que fueron creados sí le pertenecieron a un grupo determinado, el cual es suficientemente poderoso para verse protegido y a la vez legitimado para actuar de una u otra forma 
por los derechos humanos (legitimados también para mantener un determinado statu quo que favorezca sus privilegios) (Váquez, 2015).

Lo anterior dificulta la posibilidad de utilizar el discurso de los derechos humanos como herramienta política de utilidad para los «discriminados». Por ejemplo, es evidente que los derechos humanos no incluyen, per se, a los derechos de las mujeres (Bunch, 1990: 488). De otra forma, no se explica que miles de mujeres sufran diversos tipos de violencia de género y sexual sin que exista una preocupación por parte de los organismos internacionales de derechos humanos. Una razón fundamental para explicarlo es que estos derechos se basan en la distinción entre lo público y lo privado (Charlesworth, Chinkin y Wright, 1991: 623), estando, por definición, las preocupaciones de los hombres (o de ciertos hombres) en lo que se denomina el espacio público, mientras que las preocupaciones de las mujeres se encuentran en un espacio privado donde los derechos humanos no actúan (Bunch, 1990: 488).

Esta distinción no solo es suficiente para que los derechos humanos sirvan como herramienta política para mantener el statu quo, sino que también hace que sea muy difícil que puedan ser considerados como instrumento para proteger a los históricamente desprotegidos. Repensar los derechos humanos desde una perspectiva feminista será una tarea heroica (Bunch, 1990: 497).

\section{¿Cuándo fueron creados los derechos humanos? Necesidades específicas a las que respondía la creación de los derechos humanos}

Cuando la Asamblea General de las Naciones Unidas adoptó la Declaración Universal de los Derechos Humanos, muchos delegados de los diversos países representados dejaron claro que nació de las experiencias vividas en la Segunda Guerra Mundial (Morsink, 1999). Charles Malik, académico y representante del Líbano que jugó un papel crucial en su elaboración, expresó que los derechos humanos se inspiraban en la oposición de las doctrinas atroces como el nazismo y el fascismo (Morsink, 1999: 15). Además, reconocíó que la proclamación de dicha Declaración fue un milagro que se produjo por el reflejo de la Segunda Guerra Mundial, por lo que, si no se hubiese realizado en ese momento específico, se hubiese seguido trabajando en el tema por al menos diez años más (Morsink, 1999: 17).

Como consecuencia, es evidente que los derechos humanos fueron consagrados por razones políticas e históricas. En ese sentido, son un ejemplo de valores históricos o lugares comunes concebidos y desarrollados en una época específica (García, 1989: 96), lo que ya es suficiente para entenderlos como una causa política.

Así, es posible entender que los derechos humanos como discurso y causa política fueron creados como respuesta a una situación histórica específica, fijándose entonces los lineamientos generales de ciertos derechos, en primera instancia, inviolables. De la misma forma, entendiendo que en el sistema de derecho internacional tiende 
a triunfar la ley de los más poderosos (García, 1989), estos plasmaron sus preocupaciones de ese momento específico. Dicho de otra forma, los factores históricos y las condiciones sociales, políticas y culturales de un momento dado fueron variables indispensables para comprender y explicar el desarrollo previo de los derechos humanos, y también su caracterización actual (Arias, 2015: 13).

Cabe preguntarse si los derechos humanos cumplen la misma función y mantienen la misma importancia que tuvieron al momento de su creación. Actualmente, quienes tienen el poder para hacer que sus derechos humanos sean respetados son quienes raramente los ven perturbados (Burnside, 2003), mientras que quienes son recurrentemente vulnerados y necesitan la protección de ellos son los que menos posibilidades tienen de asegurarlos (Burnside, 2003: 704). Dicho problema es intrínseco a los derechos humanos.

Al constatar que el contexto específico al que respondía el establecimiento de los derechos humanos ha desaparecido, se hace necesario repensarlos y relegitimarlos (Arias, 2015: 18), ya que el consenso que existía al momento de la Declaración Universal de los Derechos Humanos se desvaneció.

¿A quién le importan ahora los derechos humanos? (Burnside, 2003: 705). Como los más poderosos se encuentran en una situación de confort con la forma actual en que se presentan, no harán nada más por ellos. Por el contrario, como los grupos usualmente vulnerados han visto que su situación no mejora con «esos» derechos humanos, tampoco se preocuparán por ellos, por lo que «esos» derechos humanos, como lugar común e histórico que se reflejó en la Declaración Universal de los Derechos Humanos, no cumplen la misma función.

Es más problemático todavía que quienes se encuentran suficientemente protegidos por estos derechos no harán esfuerzos para repensarlos, ya que hacerlo amenaza el statu quo y, con ello, sus privilegios. Debido a esto, nuevas iniciativas de la comunidad internacional relativas al establecimiento de «nuevos» derechos humanos (por ejemplo, la inmigración) están siendo cuestionados - aunque de manera incipiente- por los más poderosos. ${ }^{1}$

Esto demuestra que los derechos humanos se encuentran en peligro constante al ser valores históricos.

\section{¿Cómo fueron creados los derechos humanos? Proceso de creación y sobrelegalización}

Existen problemas inherentes relativos al proceso de creación de los instrumentos de derechos humanos (Meron, 1986), lo que implica una crítica a estos en su faceta de

1. Ana Carbajosa, «El pacto migratorio se convierte en el nuevo campo de batalla populista», El País, 30 de noviembre de 2018, disponible en https://bit.ly/2u6fJgz. 
derechos. La importancia de estas críticas es que demuestran que parte de las crisis actuales que viven los derechos humanos podrían haberse prevenido (Meron, 1986: 1), lo que denota problemáticas de legitimidad de origen.

Un primer punto relevante y criticable se encuentra tanto en la forma amplia como abstracta en que han sido redactados la mayoría de los derechos humanos (Meron, 1986: 2). Si bien esta forma de consagrarlos busca obtener la mayor cantidad de apoyo posible por parte de los distintos Estados con firmas y ratificaciones (Meron, 1986), es válido preguntarse cuál es el sentido de obtener ese tipo de apoyo, ya que es apreciable que no busca tener impactos concretos en la vida de quienes se encuentran desprotegidos. Piénsese, por ejemplo, que los tratados que impongan cualquier mecanismo de implementación concreta (tribunales o protocolos adicionales) que «tensione» la autonomía y soberanía absoluta de los Estados, tienden necesariamente a ser menos ratificados que los que se redactan de forma abstracta y general como es el caso del Pacto de Derechos Civiles y Políticos. Aquellos tratados más concretos son muchas veces denunciados (o amenazados con la denuncia o retiro unilateral) por los Estados, como ocurrió con el caso del Reino Unido en el momento en que sus fuerzas armadas efectuaron crímenes de guerra en Irak y comenzaron a ser investigados por la Corte Penal Internacional. ${ }^{2}$

Pareciera que la existencia de estos instrumentos que consagran los derechos humanos como principios abstractos y generales presupone que estos últimos serán «aterrizados» o concretizados a través de diversos tratados internacionales (Meron, 1986: 2), o bien que será la jurisprudencia de los tribunales encargados de tutelar los derechos humanos la que hará una labor interpretativa que permita delimitar lo que son estos derechos en el ámbito práctico y concreto (Meron, 1986). Ambas situaciones son problemáticas y criticables.

La primera hipótesis considera que existe una «sobrelegalización» de los derechos humanos y cada vez que se ha pretendido precisar el contenido de lo que son los derechos humanos, se ha exigido que los mecanismos de obligación y cumplimiento sean tenues, por ejemplo, que las resoluciones del tribunal que vele por su cumplimiento sean solo recomendativas (Helfer, 2002) o derechamente distintos Estados se han restado de suscribir o ratificar este tipo de tratados (Helfer, 2002: 1886). La segunda hipótesis es más difícil de practicar, ya que la creación de jurisprudencia interpretativa de los tribunales internacionales implica un proceso lento, mucho más lento que el de tribunales nacionales que deben interpretar los principios constitucionales presentes en una Carta Fundamental (Meron, 1986: 2). Además, como ya señaló, muchos de dichos tribunales carecen de mecanismos para obligar al cumplimiento de sus resoluciones.

2. Owen Bowcott, «UK calls for 'greater clarity' on ICC's new crime of aggression», The Guardian. 15 de noviembre de 2017, disponible en https://bit.ly/2tioFIi. 
Esta situación no es azarosa. La implementación del proyecto de los derechos humanos protege libertades individuales, pero también significa un costo político doméstico para cada Estado (Helfer, 2002: 1852), ya que se imponen barreras externas a su actuar. Es por eso que el discurso de los derechos humanos se plantea de manera tan abstracta. Sin embargo, esto demuestra que los derechos humanos están pensados y configurados por defecto para no ser cumplidos, identificándose un problema en su legitimidad que no radica en una crisis actual, sino en su creación misma. Así, los derechos humanos resultan impracticables como proyecto práctico.

\section{Organismos encargados de tutelar los derechos humanos: breve crítica práctica}

Como se mencionó, los derechos humanos son, entre otras cosas, un proyecto práctico que aspira a cambiar la realidad (Gallardo, 2008). Esta faceta probablemente sea la más importante, ya que implica una verdadera protección para las personas. Por lo mismo, los diversos instrumentos que consagran derechos humanos disponen de algún medio para hacerlos respetar, generalmente tribunales de justicia. Esta es la única forma de cumplir la promesa de los derechos humanos (Ariza, 2010).

Como mecanismos de defensa, estos tribunales pueden ser de variados tipos, como el Tribunal de Estrasburgo, al cual pueden recurrir directamente las personas que han visto vulnerados sus derechos humanos, y la Corte Interamericana de Derechos Humanos, a la cual solo se puede llegar si se recurre previamente a la Comisión. Ahora bien, el mecanismo de dichos tribunales de derechos humanos puede operar cuando se respeta aún un piso mínimo de derechos o garantías de las personas. Sin embargo, cuando no existe ese piso mínimo y los derechos humanos se vulneran de manera extrema (especialmente en situaciones de conflictos armados), no existen formas de protección efectivas para los afectados, quienes simplemente deberán resignarse a ver vulnerados todos y cada uno de sus derechos humanos (Burnside, 2003: 705).

Por ejemplo, a pesar de la tipificación de crímenes de lesa humanidad y crímenes de guerra, la Corte Penal Internacional actúa ex post con el objetivo de reparar, no de prevenir. Como vía fáctica, las fuerzas de paz de las Naciones Unidas solo pueden intervenir si así lo dispone el Consejo de Seguridad de la misma entidad, por lo que se trata de una decisión política de los poderosos y no protege a quienes se encuentran realmente en una posición de desprotección, como ocurrió en Ruanda. ${ }^{3}$

Esto no solo deja en evidencia que los derechos humanos son una promesa sin cumplir, sino que además son un proyecto práctico que en ningún caso se encuentra teóricamente configurado para proteger a quienes se encuentran en las situaciones

3. Chris McGreal, «What's the point of peacekeepers when they don't keep the peace?», The Guardian, 17 de septiembre de 2015, disponible en https://bit.ly/2SBCsVf. 
más extremas de desprotección (Burnside, 2003: 710). De esta forma, solo sirven cuando se parte de un piso mínimo de garantías. Todo esto hace cuestionable el proyecto práctico de los derechos humanos y reafirma la no universalidad del proyecto.

\section{Críticas al funcionamiento actual del sistema de derechos humanos}

Más allá de los problemas de legitimidad de origen que puedan tener los derechos humanos en sus diversas facetas, es innegable que actualmente están en crisis. Debido a esto es necesario que en tanto discurso como en movimiento encuentren una forma de relegitimarse, lo que de cierta forma conlleva a una refundación, y a su vez definirse en relación con los objetivos que realmente persiguen. Así, será labor de una teoría crítica del derecho poner de manifiesto estas problemáticas, refiriéndose ya no a los problemas que se presentan en el momento de creación de los derechos humanos, sino en la forma en que ellos han funcionado hasta el día de hoy.

\section{Inadecuación entre la teoría y la práctica}

Desde la Declaración Universal de los Derechos Humanos hasta ahora no solo ha habido un avance importante en la especificación de estos derechos, sino también un mayor esfuerzo por parte de la comunidad internacional (o su mayoría) por precisar su contenido y reforzar su cumplimiento. Sin embargo, ni la creación de más instrumentos internacionales y más específicos sobre derechos humanos, ni el establecimiento de mayores mecanismos para protegerlos ha significado un cambio en la vida de quienes se encuentran más resguardados (Arias, 2015: 16). En efecto, es aquí donde se puede apreciar una diferencia transversal si las discrepancias entre la teoría y la práctica de los derechos humanos se realiza desde una perspectiva interseccional: el impacto práctico de los avances teóricos o en papel solo ha afectado a quienes ya se encontraban en una posición relativamente privilegiada, mientras que quienes se encontraban realmente desprotegidos siguen estándolo, sin poder acceder a mecanismos que signifiquen una mejoría real en sus vidas. Es más, se puede señalar que los derechos humanos han servido para ignorar las problemáticas que viven los vulnerados (Pocklington, 1982), empeorando su situación. Sobre esto volveremos más adelante.

La paradoja entre la teoría y la práctica de los derechos humanos es doble. Por un lado, el discurso referencial dominante de estos derechos apela al respeto por los mismos, por y para todas las personas, proscribiendo cualquier tipo de violencia que tiende a vulnerarlos, mientras que ese mismo discurso ha servido para legitimar posiciones políticas absolutamente contrarias a los derechos humanos, como el discurso de la guerra contra el terrorismo y los discursos islamófobos, entre otros (Arias, 2015: 17). Por otro lado, se encuentra la inadecuación entre su dimensión interior 
propiamente discursiva con respecto a las efectivas condiciones sociales, políticas y culturales del momento histórico actual (Arias, 2015), siendo esta última la más evidente.

La forma en que se compone conceptualmente el discurso de los derechos humanos (derechos universales, inalienables, autoevidentes e inviolables) está tan alejada de la realidad contemporánea que hace que pierdan toda su potencialidad de instrumento de cambio para quienes lo necesitan realmente (Arias, 2015). En primer lugar, los instrumentos de los que dispone la teoría de los derechos humanos no se hace cargo de la realidad de quienes necesitan utilizar el poder emancipatorio de los derechos humanos: es ilusorio pensar que las mujeres africanas que son sometidas a la mutilación genital puedan acceder a la «justicia» que otorgan los tribunales internacionales o siquiera que puedan acercarse a ello, no solo porque es geográficamente inalcanzable, sino porque dicho sistema de instrumentos es totalmente ajeno a su cultura. Los derechos humanos fueron creados por y para occidente (Bhatta, 2009), plasman una determinada ideología y concepción sobre la justicia propia de los países hegemónicos (Bhatta, 2009: 125) y si bien tienen un discurso universalista, es bien reconocido que el disfrute práctico de los derechos humanos depende del contexto social y cultural (Bhatta, 2009: 124).

En segundo lugar, los objetivos a los que apunta el discurso de los derechos humanos tienen un sesgo político y social marcado, que se aleja de las necesidades reales de gran parte del mundo. Si bien esto se tratará con mayor detención en la sección siguiente, es evidente que la propuesta conceptual de los derechos humanos parte de un diagnóstico errado y, por lo mismo, llega a respuestas que no guardan relación con las necesidades de gran parte del mundo.

La situación descrita relacionada con la inadecuación entre la teoría y la práctica de los derechos humanos observada en dos facetas diferentes nos demuestra que el discurso de los derechos humanos vive una crisis compleja, ya que la situación real que se vive en el mundo contemporáneo amenaza sus bases de legitimidad: su discurso teórico no puede sostenerse cuando se observa la realidad de los últimos setenta años. Es urgente que se asuman las nuevas condiciones actuales (políticas y sociales) para reformular ese discurso y que se haga cargo de las condiciones actuales.

\section{Entre la homogenización y la multiculturalidad:}

\section{¿Qué buscan los derechos humanos?}

La vida contemporánea está marcada por lo que se conoce como globalización, lo que implica un proceso de homogenización que se lleva a cabo desde las pautas que el occidente hegemónico establece, ya sean económicas, culturales, sociales y valóricas, que buscan extenderse por todo el mundo como «lo correcto». Es importante precisar que entre esas pautas se encuentra el discurso de los derechos humanos. Esto, 
a su vez, lleva a otra parte de la globalización: la exacerbación de la heterogeneidad cultural (Arias, 2015: 19), es decir, que diversas identidades étnicas, religiosas y culturales buscan un lugar para reivindicarse en el mundo haciendo notar su existencia. Estos dos fenómenos son parte de la globalización y dependen de sí mismos (Arias, 2015: 19). Ciertamente, no habría necesidad de reivindicar lo «diferente» si en primer lugar no se hiciese notar que es diferente. Sobre este punto volveremos más adelante, especialmente en lo relacionado con el orientalismo y las diferencias religiosas, ya que la globalización lleva a una suerte de «choque de civilizaciones» (Arias, 2015: 20).

Entonces, es preciso preguntarse sobre el lugar en que se sitúan los derechos humanos en esta bipolaridad. Si bien hay quienes abogan por los derechos humanos como un discurso y lugar común de mediación o de puente (Bhatta, 2009: 133) por el hecho de ser inherentes al ser humano, son comprensibles para todos y trabajan en clave multicultural. También hay quienes los consideran como parte de las pautas políticas de los países occidentales y capitalistas (Arias, 2015: 22), por lo que al ser pensados y realizados en clave monocultural occidental tienen como objetivo contribuir a la homogenización.

Por ejemplo, debido a lo anterior se ha considerado por parte de la doctrina crítica de los derechos humanos que el relativismo cultural funciona como cortapisa a su universalidad, lo que no solo se debe a que muchos de los países no occidentales no participaron en la «creación» de los derechos humanos, sino también a que muchos de los que participaban en ese momento eran colonias de poderosos países occidentales, por lo que sus opiniones oficiales eran idénticas (Bhatta, 2009: 124). Esto determina la clave cultural en que fueron ideados los derechos humanos y que las prioridades de países no occidentales pueden ser distintas. Por lo mismo, su universalismo debe ser considerado en un contexto dinámico, teniendo en cuenta las significativas diferencias políticas, económicas y culturales (Bhatta, 2009: 125). Por ejemplo, muchos países del sudeste asiático se han propuesto privilegiar la paz, la seguridad y la estabilidad a través de la adopción de acuerdos de cooperación regionales y no a través de la implementación del proyecto de los derechos humanos (Bhatta, 2009). Esto tiene sentido si se consideran las señaladas diferencias, lo que hace que resulte plausible criticar las pretensiones universales de los derechos humanos.

No obstante, además es posible criticar que el discurso de los derechos humanos no toma en cuenta los diversos contextos presentes alrededor del mundo y señalar que ha contribuido a la diferenciación producto de sus objetivos homogenizantes. Žižek (2011) ha elaborado esta crítica de manera prolija al referirse a la situación de los Balcanes, quienes se «balcanizaron» (se transformaron en «lo otro») solo cuando se expusieron a los efectos de la modernización europea (y dentro de la modernización europea se puede encontrar el discurso de los derechos humanos) (Žižek, 2011: 116). En ese sentido, la intolerancia religiosa y la violencia étnica solo surgieron cuando se intentó imponer la pauta política de occidente (incluyendo el proyecto de 
los derechos humanos) y homogenizar la región. Lo mismo ocurrió con el genocidio armenio y la persecución de los kurdos llevada a cabo por Turquía: los crímenes fueron perpetrados por militares modernizadores que pretendían liberar a Turquía de su antiguo mundo y convertirla en un Estado-nación europeo (Žižek, 2011). Puede decirse lo mismo de situaciones más recientes, ya que cuando las fuerzas armadas de los Estados Unidos y el Reino Unido invadieron Irak para resguardar los derechos humanos solo lograron desestabilizar aún más la región y polarizar la zona, generando el surgimiento de diversos grupos fundamentalistas que buscaban reivindicar su identidad versus la agenda cultural que occidente estaba intentando imponer. Esto demuestra cómo los derechos humanos sirven como instrumento de homogenización y no son, de ninguna manera, un puente del diálogo entre lo homogéneo y la multiculturalidad.

También se puede aseverar que los derechos humanos tienden a condenar la otredad, lo que significa que funcionan como una herramienta homogeneizadora que permite imponer la agenda política, cultural y social de occidente. Los derechos humanos se encuentran jerarquizados (Bhatta, 2009: 125), lo que quiere decir que hay derechos más importantes que otros y, a su vez, entre ellos chocan y se superponen (Meron, 1986: 3). En esa jerarquización los valores que las democracias liberales y capitalistas consideran como fundamentales se encuentran necesariamente en la parte más alta de la pirámide, y los derechos que puedan chocar con ellos deberán ceder en su favor.

Un ejemplo son los casos que conoció el Tribunal Europeo de Derechos Humanos relacionados a la prohibición del uso del velo islámico en Francia. Catalogando el derecho de las mujeres de usar el velo islámico como parte del derecho a la libertad de culto o religiosa (Bennoune, 2007), este siempre ha debido ceder ante el secularismo (consagrado como un valor occidental), la seguridad que otros ciudadanos ven amenazada con el uso del velo islámico e incluso la igualdad de género, lo cual que es un argumento esencialmente problemático, ya que se toman medidas proteccionistas para las mujeres islámicas. Esto quiere decir que hombres y mujeres occidentales deciden que el uso del velo de manera voluntaria por parte de una mujer en un contexto en el cual no es obligatorio - Europa - atenta contra la equidad de género porque es una forma de represión contra las mujeres. Así, la libertad de expresión religiosa no puede servir como instrumento que reafirme una identidad diferente porque no representa los ideales de occidente y, al contrario, sirve como herramienta homogeneizadora de los valores occidentales.

\section{¿Para quién trabajan los derechos humanos? Instrumento de legitimación}

Un punto cúlmine de las críticas anteriores se da al observar lo que el discurso de los derechos humanos ha podido legitimar, considerando que se relaciona tanto con la 
inadecuación entre el proyecto práctico y las proposiciones teóricas, y también con la utilización del discurso de los derechos humanos como herramienta de homogeneización en la medida que legitima intervenciones contra «lo otro» para establecer (por la vía de la fuerza) lo "correcto», es decir, los valores y modelos políticos, sociales y culturales de occidente (incluso su modo de vida). Se trata, en síntesis, de una guerra en que se violan los derechos humanos de diversos pueblos que poseen identidades propias distintas a las catalogadas como aceptables por occidente en nombre de los derechos humanos.

Sobre el primer punto, se puede señalar que el discurso de los derechos humanos ha legitimado que los mismos, en cuanto derechos que consagran esferas de libertad esenciales de la persona, sean disminuidos y coartados por los Estados, lo que demuestra una vez más la inadecuación entre la teoría y la práctica. Esto ocurre porque los derechos humanos tienden a colisionar en casos determinados, lo que hace que algunos cedan en favor de otros. Piénsese, por ejemplo, en que varios países de la Unión Europea han decidido restringir algunos derechos humanos (y en ciertos casos derechamente los han suspendido) para asegurar los derechos de otros, sobre todo en casos relacionados con la paz y la seguridad de las personas (Lukic, 2006). Esto implica que mientras el discurso de los derechos humanos avanza hacia una mayor precisión y pregona no solo más derechos, sino también que tengan más fuerza vinculante, en la práctica los que guardan relación directamente con la vida de las personas se han visto disminuidos, restringidos $\mathrm{y}$, en ocasiones, suprimidos. El mayor problema es que el discurso de los derechos humanos ha servido para restringirlos y no para protegerlos.

Por otro lado, y en relación con la sección anterior, estos derechos han servido para legitimar intervenciones armadas en las cuales se han violado, en la práctica, los derechos humanos más elementales en los que pueda pensarse (y de formas extremadamente violentas). Esto ha ocurrido cada vez que los países poderosos de occidente han considerado necesario intervenir en países que consideran «distintos» porque en ellos, según su punto de vista, se estarían violando los derechos humanos. Esto ha llevado a que se imponga, por la fuerza, un régimen que consagre los valores y modos de vida propios de la sociedad occidental, bajo la justificación de que este nuevo régimen sí respetaría los derechos humanos.

En estos casos, nuevamente el discurso de los derechos humanos sirve para legitimar acciones que afectan más a ciertas identidades que otras, lo cual es apreciable desde una perspectiva interseccional. Legitiman, por ejemplo, la guerra contra el terrorismo (Agudelo, 2015), lo cual encubre ciertamente una cuota alta de islamofobia e intolerancia hacia las naciones musulmanas. También legitiman campos de detención como los del desierto en Australia, donde quienes resultan más afectados son las mujeres y los niños. Finalmente, legitiman la construcción de campos de refugiados 
en lugares que se asemejan más a campos de concentración que a cualquier forma de ayuda humanitaria, como ocurre en los campos de refugiados palestinos. ${ }^{4}$

Esta situación permite dirigir una crítica al discurso de los derechos humanos, ya que es lo suficientemente ambiguo como para permitir que con él se fundamenten proyectos que se pueden acomodar a ese discurso en el plano teórico, pero que implican llevar adelante acciones que son evidentemente contrarias a los derechos humanos. Esto repercute en su propuesta teórica y le quita un fundamento importante: la coherencia que se espera de los derechos humanos y los objetivos de este proyecto. Una teoría crítica de los derechos humanos deberá abogar necesariamente por un discurso que no permita llegar a los extremos de permitir todo tipo de actos en nombre de ellos.

\section{Conclusión}

En este trabajo se han hecho diversas críticas a los derechos humanos en varias de sus facetas y en distintos ámbitos. Nos referimos a varias facetas porque los derechos humanos son mucho más que derechos, siendo a la vez un discurso, un movimiento y un proyecto práctico. Por otra parte, nos referimos a distintos ámbitos porque se ha pretendido dirigir las críticas a distintos momentos: a los problemas de legitimidad de origen de los derechos humanos (los cuales son, por lo mismo, intrínsecos a ellos más allá de las crisis que puedan surgir en distintos momentos), y al funcionamiento actual de los derechos humanos, donde sí entran en juego las problemáticas contingentes que pueden apreciarse.

Es necesario formular las críticas a las distintas formas que adoptan los derechos humanos, es decir, ir más allá de las críticas al proyecto práctico y enfocarse también en ellos como discurso y como movimiento, ya que esto permite realizar una crítica teórica que muchas veces pasa desapercibida. En ese sentido, dirigir los cuestionamientos al discurso de los derechos humanos no solo permite comprender el origen de ellos y sus diversas mutaciones a lo largo de la historia en tanto han servido para legitimar diversas posturas en el tiempo, siempre dependiendo del contexto dominante y del statu quo, sino también que las consecuencias prácticas que ha tenido el movimiento de los derechos humanos en la vida de las personas no han sido azarosas. Al contrario, es la única repercusión que puede esperarse de ellos, lo que implica reconocer que se trata de un problema de origen, de una voluntad política estructural y no únicamente circunstancial. Es por esto que las críticas teóricas son tan importantes, ya que son las únicas que resisten la contingencia.

4. Clansy Chassay, «We have no rights and no future», The Guardian, 29 de mayo de 2007, disponible en https://bit.ly/2QBoy2v. 
Sin embargo, más allá de todas las críticas que tienden a desestabilizar la legitimidad, la propuesta y el proyecto de los derechos humanos, no se trata de desconocer la importancia de ellos. Como mencionan muchos autores, los derechos humanos como discurso significan al menos una forma de obstaculizar las actuaciones de los gobiernos, ya que imponen el deber de buscar una justificación a la hora de tomar decisiones, incluso si la justificación es insuficiente o tautológica, en cuanto a su capacidad para explicar o justificar las violaciones a los derechos humanos. Esto significa un avance porque antes podía simplemente prescindirse de cualquier tipo de justificación.

En realidad, como parte de una teoría crítica, este trabajo pretende señalar que «estos» derechos humanos no sirven (ni servirán) como instrumento de protección para quienes se han visto constantemente vulnerados y discriminados (lo cual solo puede observarse desde una perspectiva interseccional), ya que tienen problemas que van más allá de lo actual porque se refieren a su creación y, por ende, siempre afectarán su legitimidad. No obstante, esto no significa que la idea de los derechos humanos sea necesariamente criticable: un conjunto de derechos verdaderamente universales, que tome en cuenta las diferencias presentes alrededor del mundo y que tienda a restringir la soberanía de los Estados (especialmente en lo referido a su actuar violento) es un discurso que podría verse perfectamente legitimado, especialmente si dicha propuesta teórica/proyecto práctico toma en cuenta las críticas que realiza un determinado sector doctrinario, ya que solo esto le dará legitimación.

Piénsese, por ejemplo, que debido a la falta de legitimidad de origen que presentaba el conjunto de crímenes de lesa humanidad (creados por y para las preocupaciones de hombres), actualmente se están ideando nuevos crímenes de lesa humanidad, y en su creación están participando quienes usualmente habían sido dejados de lado. ${ }^{5}$ Esto, si bien no representa una solución a todos los problemas, sí demuestra un avance y una forma de relegitimar dicha institución.

Es esperable que algo similar ocurra con los derechos humanos, especialmente en un momento tan crítico como el actual, donde varios millones de personas necesitan un nuevo salvavidas al que recurrir con urgencia como ocurrió hace setenta años cuando los derechos humanos se empezaban a crear. Esto solo será posible si cambiamos el paradigma actual del sistema de derechos humanos.

\section{Referencias}

Agudelo, Martín (2015). «Una mirada alternativa de los derechos humanos a partir de Slavoj Žižek». International Journal of Žižek Studies, 9: 1-18. Disponible en https://bit.ly/39BfweS.

5. Lisa Davis, «Will the new crimes against humanity treaty protect women and LGBTI persons?», Open Democracy, disponible en https://bit.ly/2ZFIsOb. 
Anyangwe, Carlson (1996). «Human Rights: Generations, Holism and Relativity». Zambia Law Journal, 25: 81-106. Disponible en https://bit.ly/2MHXcGR.

Arias, Alan (2015). «Tesis sobre una teoría crítica de los derechos humanos». Open insight, 6 (9): 11-33. Disponible en https://bit.ly/2MGnzNs.

Arias, Alan y Fabiola Ponte (2015). «Hacia la relegitimación del discurso de los derechos humanos en América Latina». El Cotidiano, 194: 31-40. Disponible en https:// bit.ly/39u6ZtQ.

ArizA, Rosembert (2010). «Los derechos humanos en América Latina: una promesa sin cumplir». Diálogo Político, 27 (4): 73-90. Disponible en http://www.corteidh. or.cr/tablas/r26354.pdf.

Bennoune, Karima (2007). «Secularism and Human Rights: A Contextual Analysis of Headscarves, Religious Expression, and Women's Equality under International Law». Columbia Journal of Transnational Law, 45 (2): 367-426. Disponible en https://bit.ly/2MKoXeW.

BhatтA, Diwakar (2009). "Cultural Relativism: An Examination of Asian Values as a Shield against Human Rights Violations in East Asia». NJA Law Journal, 3 (1): 123-134. Disponible en https://bit.ly/2F8GXOY.

Bunch, Charlotte (1990). «Women's Rights as Human Rights: Toward a Re-Vision of Human Rights». Human Rights Quarterly, 12 (4): 486-498. Disponible en https:// bit.ly/2Q8A4DA.

Burnside, Julian (2003). «Who Cares about Human Rights». UNSW Law Journal, 26 (3): 703-716. Disponible en https://bit.ly/2F8djcF.

Charlesworth, Hilary, Christine Chinkin y Shelley Wright (1991). "Aproximaciones Feministas al Derecho Internacional». The American Journal of International Law, 35: 613-645.

FACIO, Alda (2000). «Hacia otra teoría crítica del Derecho». En Lorena Fries y Alda Facio (editoras), Género y Derecho (pp. 15-44). Santiago: LOM.

Gallardo, Helio (2006). Derechos humanos como movimiento social. Bogotá: Editorial Desde Abajo.

Gallardo, Helio (2008). «Notas sobre derechos humanos y políticas públicas». Quito: Programa Andino de Derechos Humanos. Disponible en http://bit.ly/36iMy1e. García, Mauricio (1989). «Teoría y práctica de los Derechos Humanos». Revista Facultad de Derecho y Ciencias Políticas UPB, 83: 94-113. Disponible en https://bit. ly/39qOWF8.

Helfer, Laurence (2002). «Overlegalizing Human Rights: International Relations Theory and the Commonwealth Caribbean Backlash against Human Rights Regimes». Columbia Law Review, 102: 1832-1911. Disponible en https://bit.ly/2u8z7mt. Howse, Robert (2002). "Human Rights in the WTO: Whose Rights, What Humanity? Comment on Petersmann». European Journal of International Law, 13 (3): 651-659. Disponible en https://bit.ly/37ljUwu. 
Lukic, Tatjana (2006). «Борба Против Тероризма На Нивоу Европске Унијеи Основна Људска Права И Слободе». Zbornik Radoba, 40: 447-462.Disponible en http://bit.ly/2QgEWGJ.

Meron, Theodor (1986). Human Rights Law-Making in the United Nations. Cambridge: Cambridge University Press.

Morsink, Johannes (1999). The Universal Declaration of Human Rights: Origins, Drafting, and Intent. Filadelfia: University of Pennsylvania Press.

Pocklington, Thomas (1982). «Against inflating human rights». Windsor Yearbook of Access to Justice, 2: 77-86

SмIтн, Barbara (2000). Home Girls: A Black Feminist Anthology. Nueva Jersey: Rutgers University Press.

Symington, Alison (2004). «Interseccionalidad: una herramienta para la justicia de género y la justicia económica». AWID-Revista Género y Derechos, 9: 1-8. Disponible en https://bit.ly/2F9yBXy.

VÁqueZ, Luis (2015). «¿El discurso anticapitalista de los derechos humanos?». El Cotidiano, 194: 57-64. Disponible en https://bit.ly/3568rzm.

Wiesen, Blanche (1992). «Eleanor Roosevelt and Human Rights: The Battle for Peace and Planetary Decency». En Edward Crapol, Women and American Foreign Policy: Lobbyists, Critics, and Insiders (pp. 98-118). Nueva York: Greenwood Press.

ŽıžEK, Slavoj (2011). «En contra de los derechos humanos». Suma de Negocios, 2 (2): 119-127. Disponible en https://bit.ly/2F4Uib6.

\section{Sobre la autora}

Magadalena Abraca Lizana es egresada de la Facultad de Derecho de la Universidad de Chile y coordinadora de asistentes del curso Teoría Crítica del Derecho. Su correo electrónico es magdabarcalizana@gmail.com 